\title{
Desarrollo neuroembriológico: el camino desde la proliferación hasta la perfección
}

\author{
Cristhian Eduardo Hernández-a \\ FLÓREZ \\ Universidad Industrial de Santander, Colombia \\ Mónica Andrea Beltrán ${ }^{b}$ \\ Universidad Industrial de Santander, Colombia \\ Gustavo Adolfo Contreras ${ }^{c}$ \\ Universidad Industrial de Santander, Colombia
}

\footnotetext{
a Médico y cirujano. Miembro del Grupo de Genética Humana, Universidad Industrial de Santander, Bucaramanga, Colombia.

b Médica especialista en Ginecoobstetricia y Perinatología, Departamento de Ginecología y Obstetricia, Hospital Universitario de Santander-Universidad Industrial de Santander, Bucaramanga, Colombia.

c Médico especialista en Genética Médica. Especialista en Bioética. Departamento de Pediatría, Hospital Universitario de Santander-Universidad Industrial de Santander, Bucaramanga, Colombia.
}

Financiación: Este manuscrito fue realizado y financiado con recursos propios de los autores.

Cómo citar: Hernández-Flórez CE, Beltrán MA, Contreras GA. Desarrollo neuroembriológico: el camino desde la proliferación hasta la perfección. Univ. Med. 2018;59(3). doi: https://doi.org/ $10.11144 / J a v e r i a n a . u m e d 59-3$. dneu

\section{RESUMEN}

El desarrollo neurológico humano requiere una serie de pasos que permitan orientar, regular y diferenciar los diversos componentes cerebrales, para así garantizar, de una manera bastante precisa, la correcta organización y funcionamiento de las estructuras neuronales. La neurogénesis está clásicamente dividida en cuatro etapas consecutivas: proliferación, migración, diferenciación y maduración. En los humanos, estas ocurren desde la tercera semana de gestación hasta la vida adulta y precisan de un complejo grupo de paquetes genéticos, así como de algunos factores asociados, que se han ido descubriendo gracias a los avances en la biología molecular. El artículo es una revisión acerca del desarrollo neuroembriológico humano y los componentes genéticos más relevantes encontrados en la literatura.

\section{Palabras clave}

neurogénesis; desarrollo embrionario y fetal; migración celular; diferenciación celular; plasticidad.

\section{ABSTRACT}

The human neuronal development requires a number of concrete steps which lead to orientation, regulation and differentiation of several brain components. They must be done to guarantee, in a very precise way, the correct organization and functioning of the neuronal structures. Neurogenesis is commonly divided into four consecutive stages: proliferation, migration, differentiation and maturation. In humans, those stages take place since the third week of prenatal life until the adult life. They also require a complex group of genetic packs and associated molecular factors, most of which have been recently discovered by the molecular biology technology. A review was made about the human neuronal and embryological development and the most relevant genetic components described by the literature so far.

Keywords

neurogenesis; embryonic and fetal development; cell migration; cell differentiation; plasticity. 


\section{Introducción}

El cerebro humano se considera el órgano más misterioso, complejo y estructurado de todos los sistemas biológicos, un modelo de lo que podría ser calificado como muestra de la perfección evolutiva. Su organización, al igual que todos los detalles de su formación, sigue siendo objeto de variadas hipótesis y discusiones; en tanto que la ciencia actual aún no ha podido descifrar del todo los secretos a su alrededor. Pese a ello, algunos avances se han llevado a cabo, y parte de los procesos de neuroformación comienzan a ser dilucidados, lo que ha permitido incluso la realización de modelos computarizados y la descripción de variables de desarrollo citogenético en hongos, animales y humanos $(1,2)$.

Comprender el desarrollo del principal elemento para el funcionamiento orgánico es uno de los principales objetivos de las investigaciones actuales, ya que dilucidando las etapas y componentes moleculares se pueden establecer estrategias futuras tanto para el diagnóstico como para el tratamiento de patologías neurológicas. Este entendimiento cobra especial importancia para quienes reconocen que una alteración en la génesis del sistema nervioso central (SNC), además de ser a menudo incompatible con la vida, se presenta con una amplia gama de manifestaciones clínicas, la cual cubre desde una leve dificultad de aprendizaje hasta verdaderas malformaciones anatómicas y severos cuadros convulsivos (3).

\section{Neurogénesis}

En un estudio clásico de Dobbing y Sands (4) en el que se compararon 139 cerebros humanos normales de entre 10 semanas de gestación y 7 años posnatales, más 9 de adultos, se demostró cómo desde la etapa prenatal de esta especie hay una gran actividad en el desarrollo del SNC. Este proceso se extiende, en condiciones normales $(5,6,7)$, de manera vertiginosa durante los primeros tres o cuatro años de vida para seguir el crecimiento, aunque menos acelerado, hasta la edad adulta. De tal manera, el cerebro humano ya ha alcanzado hacia los seis años, cerca del $90 \%$ de su volumen final (8). El índice de celularidad encontrado en las muestras mediante análisis con cuantificación de ADN (unidades de ADN/ gramo de tejido) por el método de Zamenhof (4) debe suponer el desarrollo de una población cerebral adulta cercana a los cien mil millones de neuronas, comunicándose entre ellas y cada una con más de mil de otras neuronas, lo que genera alrededor de 60 billones de conexiones neuronales o sinapsis (8).

Para ello se requiere una serie de procesos en que interactúan múltiples genes, los cuales median desde la proliferación celular hasta la creación de verdaderos circuitos nerviosos. Estos llegan a ser tan complejos que se estima que cerca de un tercio del genoma humano está destinado solo para garantizar el correcto desarrollo, mantenimiento y comunicación de las estructuras nerviosas (9). Según la literatura, el desarrollo del SNC se puede dividir en cuatro grandes periodos (7) consecutivos:

Proliferación: semanas 1 a 7 .

Migración: semanas 8 a 15 .

Diferenciación: semanas 16 a 26.

Maduración: semanas 27 a adultez.

\section{Proliferación}

Una vez ocurridas las fases básicas del desarrollo embrionario (progénesis, fecundación y segmentación), en la segunda semana de gestación el embrión consiste en una simple estructura bilaminar que se conforma de una capa superior por células epiblásticas y una inferior de células hipoblásticas. Es entonces cuando se da comienzo al proceso de gastrulación, en el cual las células epiblásticas se diferenciarán en tres capas de células madre que formarán directamente los diversos tejidos y sistemas orgánicos; mientras tanto, las células hipoblásticas formarán las estructuras extraembrionarias como la placenta (8). Las células epiblásticas de la capa más basal formarán el endodermo que originará las estructuras viscerales y del sistema respiratorio, en tanto que la nueva capa intermedia - 
que se conoce como mesodermo- formará las estructuras vasculares y osteomusculares. La capa más superficial, conocida como ectodermo, se diferenciará entonces en ectodermo epidérmico —que dará origen a estructuras como piel, uñas y glándulas sudoríparas- y en neuroectodermo -que creará las estructuras propias del SNC(8). En la figura 1 se muestra la organización de dichos tejidos (8).

\section{Figura 1}

Principales eventos de la gastrulación entre los días 13 y 20 de gestación con la ubicación espacial de las capas embrionarias. A) Vista superior de la migración de las células epiblásticas hacia el nodo primitivo, dirigida en el sentido que muestran las flechas. B) Corte transversal del embrión. C) Migración celular por debajo de la capa superior y en sentido rostral para conformar las nuevas capas de endodérmica y mesodérmica. D) Las células que migran paralelas a la línea media envían señales moleculares que inducen al epiblasto circundante y delimitado por el cuadro rojo a convertirse en células neuroprogenitoras. Posteriormente las células del nodo primitivo lanzan otra secuencia de señales que indicarán los subtipos en que se diferenciarán estas células neuroprogenitoras

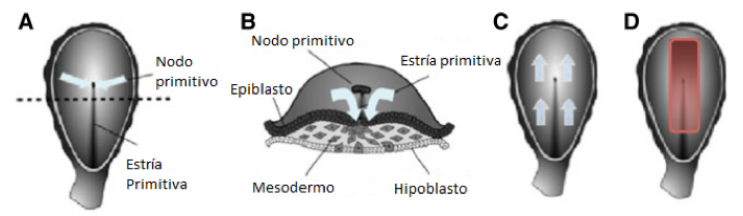

Fuente: tomado y modificado de The Basics of Brain Development (figura 5) (8). Open Access en http://springerlink.com

Al principio de la tercera semana, en la cara dorsal del disco embrionario aparece una línea media de células neuroprogenitoras en la llamada estría primitiva, la cual se alarga desde el extremo caudal para formar el nódulo primitivo al llegar al extremo craneal. Este nódulo actuará como un centro de señalización molecular que mediará las expresiones génicas de las células migratorias $(8,9)$. De igual manera, aquellas células dejarán a su paso una secreción proteica entre sí mismas y las que permanecen en la región media de la capa superior del epiblasto, la cual estimula los receptores de membrana celulares induciendo su diferenciación a células neuroprogenitoras. En este punto se da por terminada la etapa de gastrulación, a la vez que la zona donde se han concentrado las células neuroprogenitoras se conocerá como placa neural $(8,10)$.

Hacia el día 21 de gestación aparecen dos surcos en los bordes laterales de la placa neural que contienen las células neuroprogenitoras y se amplían progresivamente hasta adquirir una disposición tubular; es entonces cuando se forma la primera estructura nerviosa propiamente dicha: el tubo neural $(8,10)$. La fusión de los bordes laterales del tubo neural se origina en el centro de la estructura y lentamente se extiende hacia los extremos rostral y caudal, formando los neuroporos anterior (rostral) y posterior (caudal), los cuales de igual manera se cierran hacia los días 25 y 27, respectivamente. Las células de la capa en la línea media se reorganizarán hacia el centro del tubo neural, en una cavidad que formará los ventrículos cerebrales en la llamada zona ventricular $(Z V)(8,11,12)$. Aquellas células que se dispongan hacia la zona caudal conformarán el tallo cerebral y la médula espinal (8).

En cuanto a las células que se disponen hacia una ubicación cefálica, expondrán el llamado primordio telencefálico, el cual va a conformar estructuras vitales como la corteza cerebral, el bulbo olfatorio, el hipocampo, los ganglios basales y la amígdala (13), y se compone en su totalidad de células neuroepiteliales en división. Tal estructura es conocida como la matriz o el epitelio germinal, un epitelio homogéneo seudoestratificado que se divide de manera radial, expansiva y simétrica $(7,10)$. Este se considera un punto especialmente crítico en los humanos, pues es cuando se comienza a diferenciar de otras especies como los roedores, mediante hallazgos anatómicos como un gran engrosamiento de su superficie telencefálica $(14,15)$.

En algún punto cerca del día 33 de gestación, estas células realizan una división asimétrica que marca el comienzo propio de la neurogénesis. Con el inicio de la neurogénesis ocurre también un proceso conocido como determinación, el cual corresponde únicamente a la estabilización genotípica de las células precursoras, mediante el switch genético o los recursos de activación 
y desactivación de bases génicas conforme a su programación hereditaria (16). En estos casos, una célula hija permanece como futura progenitora o célula pluripotencial (17); mientras la otra está destinada a sufrir diferenciación (caracterización fenotípica) para convertirse en una neurona o en una célula de la glía $(7,12,15)$. Es acá donde se comienzan a formar, aunque de manera muy rudimentaria, todo el material para las estructuras corticales y subcorticales, así como las vías sinápticas (8).

Múltiples genes son entonces los encargados de regular estos complejos procesos de determinación y diferenciación con subsecuente proliferación celular. Algunos de ellos son el Forhead Box G1 (FoxG1), LIM Homebox 2 (Lhx2), Paired Box 6 (Pax6) y Empty Spiracle Homologue 2 (Emx2), además de ciertos precursores intermedios de diferenciación como los factores de transcripción Tbr2, neurogenina 2 y Cutlike Homebox 1 y 2 (Cux 1 y 2) (13,14). Es en este instante cuando desempeñan un rol vital los sistemas de circulación, oxigenación, interacciones metabólicas y respuestas inmunes, para garantizar un armónico trabajo que garantice la ausencia de fallas estructurales, complicaciones o malformaciones (7).

A partir de la formación básica de las estructuras del SNC, se comienza un proceso expansivo de las estructuras nerviosas primordiales, pasando el embrión de tener 3-5 $\mathrm{mm}$ de largo hasta alcanzar los 27-31 $\mathrm{mm}$ al final de la semana 8. Adicional a ello, se comienzan a materializar algunas estructuras más específicas o las llamadas vesículas cerebrales primarias anterior, media y posterior, o prosencéfalo, mesencéfalo y romboencéfalo, respectivamente. La primera se dividirá luego en telencéfalo y diencéfalo, y la tercera en metencéfalo y mielencéfalo; aquellas 5 vesículas originan la totalidad de estructuras del SNC (8). En la figura 2 se muestran, aunque de manera esquemática, las estructuras originadas a partir de las vesículas primordiales.

\section{Figura 2}

Evolución de las estructuras anatómicas del sistema nervioso central a partir de las vesículas primordiales, organizadas según su distribución espacial de anterior-rostral a posterior-caudal. En negrita de la tercera columna, las cinco vesículas secundarias

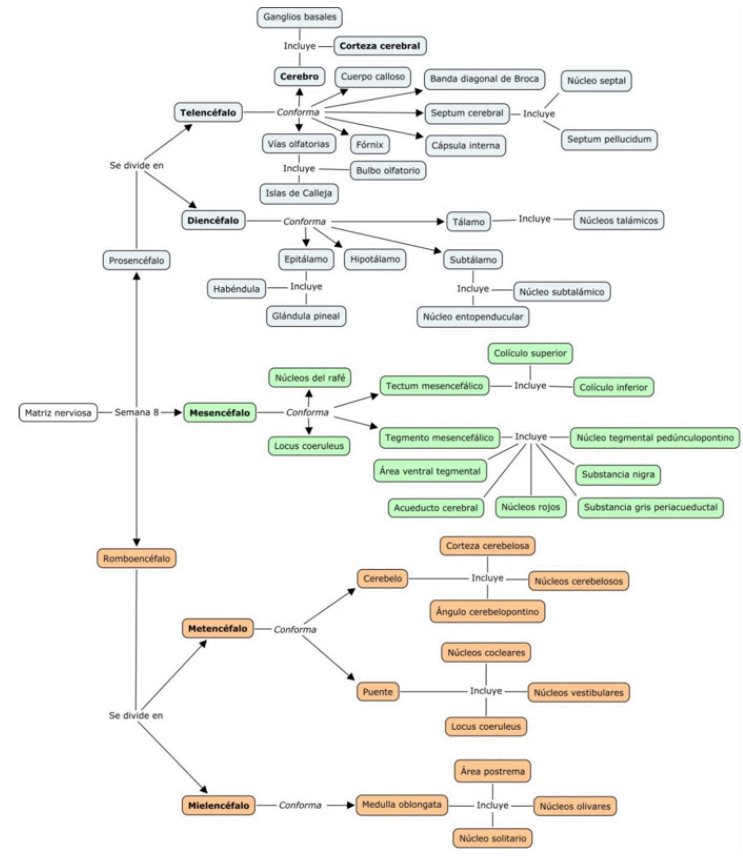

\section{Migración}

El desarrollo cortical se realiza en un llamado "de adentro para afuera", lo cual indica que las estructuras subcorticales o internas del encéfalo empiezan primero y las superficiales después. Las células que nacen en las regiones proliferativas de la ZV y la zona subventricular (ZSV) — aquellas que no están adheridas a la superficie ventricular - migran hacia la placa cortical guiándose por las proyecciones basales dispuestas durante expansión radial de las células de la glía $(1,2,10,11,12,18,19,20,21,22)$. Las primeras capas ya establecidas son observables por histología a partir de la semana $12(7,8)$; sin embargo, a partir de la semana 17, estas pueden distinguirse mediante imagenología con el uso de la resonancia nuclear magnética (10).

$\mathrm{Se}$ cree que las células progenitoras procedentes de la ZV constituyen el recurso 
principal para las capas más basales de la corteza ( $\mathrm{V}$ y VI); mientras que las de la ZSV corresponden sobre todo para las capas superficiales (IV, III y II). Pese a ello, hay alguna evidencia que sugiere la presencia de células de la ZSV en todas las capas corticales $(7,12)$. De igual manera, algunos de los sets genéticos, como el Pax6 y el Nr2el o Tlx, pueden controlar la proliferación de las células progenitoras de la ZV durante el establecimiento y la expansión de la ZSV. Mutaciones a nivel del Pax6 se relacionan con disminución neuronal de las capas superficiales de la corteza cerebral -aunque con normalidad de las capas basales-; a su vez, el set mutante de Nr2el se relaciona con una disminución en el tamaño de la ZSV, con menor proliferación y una aparición de las capas superficiales neocorticales uno o dos días antes de lo normal (12).

De esta etapa y por la correcta dirección genética depende que, por ejemplo, la neocorteza se encuentre perfectamente dividida en distintas áreas, cada una con neuronas específicas estructural y funcionalmente $(8,12)$. Por ello existen algunos mecanismos moleculares, como las GTPasas de las familias Rho y Rab, que regulan la organización citoesquelética de las células migratorias, así como los elementos adhesivos $\mathrm{y}$ otros factores esenciales para una correcta estructuración neocortical durante el desarrollo embriológico $(20,23,24)$. Dicha regulación se realiza mediante la expresión de algunas proteínas como la Racl y $\mathrm{Cdc} 42$, las cuales interactúan directamente con los mecanismos de la translocación nuclear, generando cambios dinámicos en la disposición de los microtúbulos y actina citoplasmáticos. Algunos estudios en roedores parecen determinar que deficiencias en la Racl son responsables de severas malformaciones anatómicas corticales, por lo que debe ser una proteína decisiva para la renovación, supervivencia y migración neuronal. Asimismo, alteraciones en la $\mathrm{Cdc} 42$ generan una pérdida en la expresión de moléculas como las Ecaderinas, $\beta$-cateninas y el complejo Par, vitales para mantener estables las uniones celulares y permitir la replicación mitógena (24).
Aunque se considera que este proceso obedece a esquemas geométricos en el modelo espaciotemporal (25), también han sido propuestas diversas fórmulas matemáticas basadas en patrones multicelulares de migración y en las cuales tienen en cuenta factores como las fuerzas de repulsión intercelular, el efecto adhesivo en el arrastre migratorio y los sistemas estocásticos de cada célula en particular (23). Por otro lado, algunos elementos intrínsecos —como fallas en la expresión genética- o extrínsecos - como la exposición materna a cocaínapueden interferir de manera importante en el proceso de migración neuronal, algo demostrado en modelos roedores y por lo que todos los defectos neurogénicos de migración deberían ser considerados de naturaleza multifactorial $(26,27)$.

De todas maneras, no se debe olvidar que las neuronas poseen ciertos recursos de compensación en caso de que algunos de sus mecanismos genéticos fallen. Uno de estos es la plasticidad celular, definida como la capacidad para cruzar los límites entre linajes celulares en condiciones microambientales adecuadas $(12,17,28)$. En neuroembriología, el término plasticidad se aplica en situaciones durante las cuales hay una inexpresión (o sobreexpresión) específica de cierta señal molecular de determinación o diferenciación. Así se ha revelado la existencia de algunos mecanismos de regulación y contrarregulación entre las propias proteínas, dependiendo del comportamiento de cada una según la manifestación de un gen diferente.

En condiciones normales, algunos genes se pueden expresar más que otros; una situación que tiende a variar también entre individuos, por lo que no se ha llegado a comprender en su totalidad los factores determinantes del desarrollo neuronal. Sin embargo, por ahora se reconocen algunos otros elementos de los que dependen estos mecanismos, como la presencia o ausencia de factores extrínsecos como las neurotropinas o el factor de crecimiento nervioso (7), el Sonic Hedgehog (SHH), los factores de crecimiento fibroblástico y las proteínas morfogenéticas óseas. También se 
encuentran relacionados algunos de los genes ya mencionados como el Lhx2, FoxG1, Emx2 y Pax6, expresados a través de las paredes laterales del telencéfalo; los dos primeros genes se orientan hacia la línea media dorsal, y los restantes, hacia la ventral, limitando la expansión celular (12). Ante la ausencia del $\operatorname{Lh} \times 2$, se observa una dramática expansión de las células procedentes de la ZV más allá de la línea media dorsal, en tanto que con el déficit de FoxG1 hay una inespecificidad de progenitores neocorticales con expansión de las áreas hipocampales (12).

Por otro lado, dos de los genes, el Pax6 y el Emx2, han demostrado tener un rol significativo durante la determinación de las regiones corticales, al mantener cierto equilibrio entre sus expresiones proteicas (12): altas concentraciones de Pax6 con bajas de Emx2 inducen la diferenciación hacia neuronas motoras; en cambio, concentraciones inversas estimulan la sensibilidad visual. Estudios en roedores mutantes han demostrado que si se bloquean las vías del Pax6, hay una hipotrofia de las áreas somatosensoriales y motoras, a la vez que se amplifica la población de neuronas visuales, evento que ocurre de manera contraria cuando se bloquean las del Emx2. Dichos cambios se evidencian en la figura $3(8,29)$. Sin embargo, cuando se bloquean ambos genes, el resultado es una drástica reorganización sensoriomotora de toda la neocorteza con ausencia de varias líneas corticales y la expansión de los progenitores ventrales a través de todo el telencéfalo dorsal $(8,12)$.

\section{Figura 3}

Efectos experimentales de bloqueo selectivo de los genes Emx2 y Pax6 en ratones mutantes. Nótense las diferencias en la división cortical conforme se bloquea cada uno de los sets genéticos

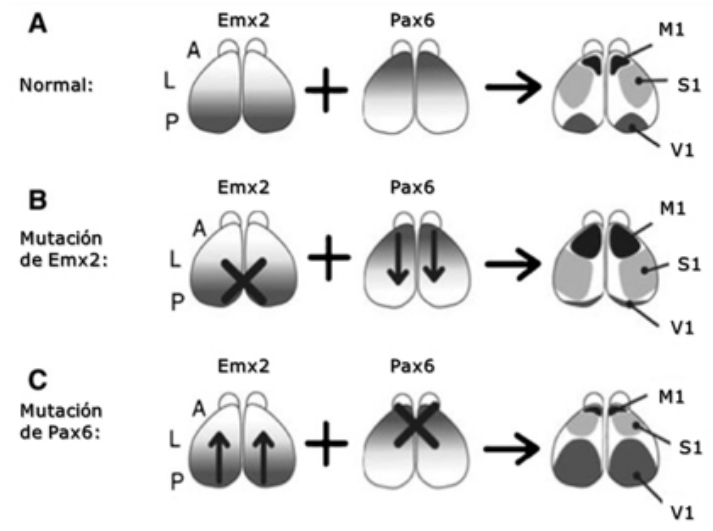

A: anterior. L: lateral. P: posterior. M1: corteza motora. S1: corteza sensitiva. V1: corteza visual. Fuente: tomado y modificado de The

Basics of Brain Development (figura 7) (8).

Open access en http://springerlink.com

Dado esto, son muchas las patologías que pueden originarse si ocurriera algún fallo durante este complejo proceso de migración neuronal. La esquizencefalia, lisencefalia, agiria, macrogiria, paquigiria, microgiria, polimicrogiria y heterotipia se consideran las alteraciones de la migración más comunes y su clasificación depende de los hallazgos morfológicos $(10,24,27,30)$. Sin embargo, gracias a los mecanismos de compensación y la plasticidad neuronal, su espectro clínico puede variar desde la incompatibilidad con la vida o severos retrasos del desarrollo hasta pasar inadvertidas y encontrarse como hallazgos incidentales durante la vida adulta $(31,32)$.

\section{Diferenciación y maduración}

La completa maduración neuronal requiere una especialización celular total, conexiones nerviosas definitivas y refuerzos mecánicos tisulares suficientes, un proceso que se necesita desde las últimas semanas prenatales hasta los primeros años de vida para la mayoría de 
especies; incluso ello se observa en la vida adulta humana $(4,7)$. La diferenciación celular es entonces un proceso en el cual las células precursoras, siguiendo los patrones que le han sido determinados genéticamente, adquiere un verdadero cambio fenotípico hacia tipos celulares específicos o especializados (17). En este periodo sucede, además, una verdadera interconexión funcional de los diversos circuitos neuronales preformados durante la migración, como el que se requiere para la integración de las neuronas gabaérgicas con las glutamatérgicas $(7,12,22,26)$. Se estima que, tras la semana 34 de gestación, el cerebro fetal humano está conectando alrededor de 40.000 uniones sinápticas nuevas por segundo (10).

A diferencia de los demás tipos de células madre, las células neuroepiteliales son heterogéneas y parecen tener cierta restricción respecto a la cantidad y variedad de hijas en las que pueden proliferar. Dado esto, y a medida que avanza el desarrollo cortical, la piscina celular de la ZV y la ZSV empieza a reducirse paulatinamente, presentando además cambios como la pérdida de polaridad apical-basal, que indican la transición de naturaleza proliferativa a neurogénica $(7,30)$. Algunos otros factores como la mayor duración de los ciclos celulares y la horizontalización de las divisiones también pueden indicar este cambio de comportamiento. Cabe anotar que esta fase de desarrollo ha sido relacionada con la expresión o restricción de varios set genéticos y factores de transcripción como el Tbr2 (7). En la figura 4 se muestra el patrón de desarrollo telencefálico, se resumen los periodos y se aclaran algunos conceptos (7).

\section{Figura 4}

Patrones del desarrollo telencefálico. Es de anotar un incremento en la complejidad de las líneas celulares conforme evoluciona el desarrollo de la neocorteza. Al comienzo de la neurogénesis las estructuras son sostenidas por los pericitos a la vez que es infiltrada por células neuroprogenitoras que se convertirán en neuronas o células de la glia y se acumulan dentro de la zona ventricular. Una segunda zona de proliferación se forma en la zona subventricular. En la segunda y tercera fases las neuronas migran y se diferencian dentro de las capas neocorticales, estableciendo conexiones anatómicas y funcionales. En la fase 4 ocurre una gliogénesis y en el telencéfalo dorsal y ventral con incremento de la migración gabaérgica hacia la corteza. Durante la maduración puede haber remodelación vascular y maduración con reintegración sináptica

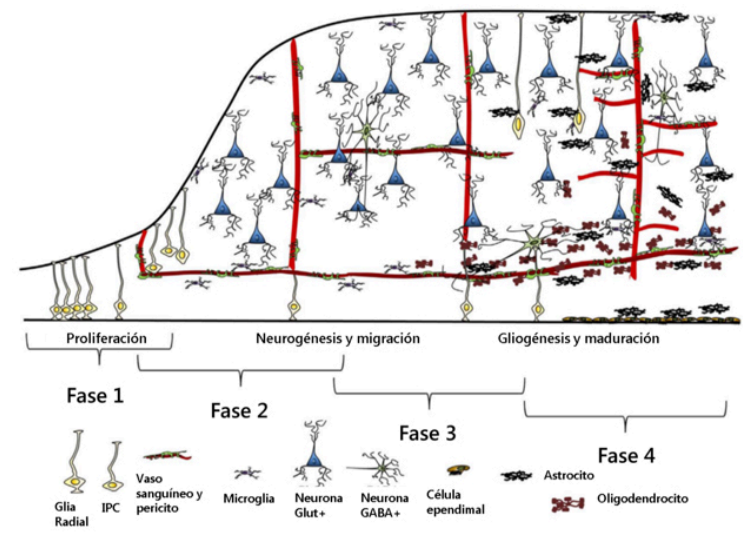

Fuente: tomado y modificado de The Long and the Short of It: Gene and Environment Interactions During Early Cortical

Development and Consequences for Long-

Term Neurological Disease (figura 4) (7).

Free access article by Frontiers Media S. A.

En cuanto a la mielinización, esta es necesaria para garantizar tanto el aporte energético como el metabolismo neuronal, y se realiza de manera conjunta con la proliferación y diferenciación de los oligodendrocitos. La mielinización de la sustancia blanca comienza uno o dos meses antes del nacimiento y se extiende hasta los nueve meses posnatales. El proceso recorre acorde a la maduración de los circuitos funcionales, por lo que las fibras son mielinizadas en sentido posterior-anterior, siguiendo primero las fibras de 
las áreas sensitivas, luego las motoras y al final las asociativas (10).

Por último, se considera que el cerebro humano maduro ingresa en una fase de degeneración fisiológica, en la que pierde cerca del $40 \%$ de sus cuerpos neuronales respecto a los que poseía al final de la etapa prenatal. Esto se acompaña con un adelgazamiento progresivo no lineal de las capas corticales parietales y dorso frontal desde la adolescencia hasta los 45 años. En condiciones normales, durante la quinta década de la vida, la materia blanca no sufre modificaciones; pero se empieza a observar una degeneración de la materia gris, sobre todo la del lóbulo temporal izquierdo, y que se puede relacionar con las alteraciones del lenguaje que se suelen empezar a observar en aquella edad. De todas maneras, la información disponible es apenas sugestiva e incipiente, por lo que se requieren más y mejores estudios para confirmar dichos patrones y entender la naturaleza tanto del desarrollo como de la degeneración neuronal (10).

\section{Conclusiones}

La neuroembriología es, sin duda, un área fascinante y de rápido desarrollo. De la mano con la genética y la biología molecular, se han ido revelando poco a poco las piezas que arman el rompecabezas del desarrollo cerebral. Esto al tiempo que se vislumbran, cada vez más cerca, posibilidades reales de pronóstico y tratamiento para las malformaciones del SNC. Los avances en las ciencias básicas enlazados con la clínica establecerán el camino para el manejo de las enfermedades relacionadas con este sistema.

Ahora se conocen algunos genes específicos, como los mencionados Rho, Rab, Pax6, Nr2el y $E m x 2$, que poseen funciones particulares en la organización neuronal. También se están esbozando las líneas de migración y se han transcrito varios de los procesos que indican la propia diferenciación del epitelio germinal. Sin embargo, todavía falta una gran cantidad de datos que permitan predecir, si no es que manipular, los circuitos de información interneuronales y, en general, los mecanismos de proliferación y diferenciación celulares. Por tanto, el entendimiento molecular del proceso de desarrollo neuronal brindará verdaderas herramientas de prevención, diagnóstico y tratamiento para estas patologías $(31,32,33)$.

$\mathrm{Si}$ bien se puede pensar que al intentar comprender al cerebro humano se estaría buscando desentrañar los misterios propios de la conciencia y la composición de los pensamientos, se debe considerar que es necesario conocer acerca de su desarrollo. Aquello para poder ampliar el campo de acción de la medicina como herramienta de esperanza y curación en aquellas familias con riesgo genético o niños con daño cerebral reparable. Todavía queda mucho por estudiar, patrones que explicar y genes que descubrir; pero como dice una frase anónima: "si el cerebro humano fuese tan simple que pudiésemos entenderlo, entonces seríamos tan simples que no podríamos entenderlo".

Hoy en día la neuroembriología es un campo en desarrollo. Esta revisión nos abre la puerta para proponer estudios basados no solo en la formación estructural del SNC, sino en indagar los mecanismos moleculares y vías de señalización celular. Teniendo en cuenta que tenemos un campo de acción clínico, es interesante establecer una línea de investigación que pueda unir tanto el área de ciencias básicas como clínica, campo que actualmente estamos desarrollando en conjunto con la participación de diferentes especialidades de nuestra institución.

\section{Referencias}

1. Keays DA. Neuronal migration: Unraveling the molecular pathway with humans, mice, and a fungus. Mamm Genome. 2007;18:425-30.

2. Setty Y, Chen C, Secrier M, Skoblov $\mathrm{N}$, Kalamatianos D, Emmott S. How neurons migrate: a dynamic in-silico model of neuronal migration in the developing cortex. BMC Syst Biol. 2011;5:154. 
3. Sastre E. Alteraciones de la migración neuronal y epilepsia: Factores implicados, terapias actuales y perspectivas de futuro [Internet]. [Tesis de maestría]. Salamanca: Universidad de Salamanca; 2009. Disponible en: ht tp://hdl.handle.net/10366/74553.

4. Dobbing J, Sands J. Quantitative growth and development of human brain. Arch Dis Child. 1973;48:757-67.

5. Johnston M, Ishida A. Plasticity and injury in the developing brain. Brain Dev. 2009;31,1-10.

6. McQuillen P. Effects of congenital heart disease on brain development. Prog Pediatr Cardiol. 2010;29:79-85.

7. Stolp H, Neuhaus A, Sundramoorthi R, Molnár Z. The long and the short of it: Gene and environment interactions during early cortical development and consequences for long-term neurological disease. Front. Psychiatry. 2012;3:50.

8. Stiles J, Jernigan TL. The basics of brain development. Neuropsychol Rev. 2010;20:327-48.

9. Dimecky SM, Kim JC. Molecular neuroanatomy's "Three Gs": A primer. Neuron. 2007;54:17-34.

10. Marsh R, Gerber AJ, Peterson BS. Neuroimaging studies of normal brain development and their relevance for understanding childhood neuropsychiatric disorders. J Am Acad Child Adolesc Psyquiatry. 2008;47:1233-51.

11. Marín O, Valiente M, Ge X, Tsai LH. Guiding neuronal cell migrations. Cold Spring Harb Perspect Biol. 2010;2:a001834.

12. Molyneaux BJ, Arlotta P, Menezes JRL, Macklis JD. Neuronal subtype specification in the cerebral cortex. Nature Rev Neurosci. 2007;8:427-37.

13. Corbin J, Gaiano N. Regulation of neural progenitor cell development in the nervous system. J. Neurochem. 2008;106:2272-87.

14. Bystron I, Blakemore C, Rakic P. Development of the human cerebral cortex: Boulder Committee revisited. Nature Rev Neurosci. 2008;9:110-22.

15. Barkovich AJ, Guerrini R, Kuzniecky RI, Jackson GD, Dobyns WB. A developmental and genetic classification for malformations of cortical development: update 2012. Brain. 2012;135:1348-69.

16. Lim HN, Van Oudenaarden A. A multistep epigenetic switch enables the stable inheritance of DNA methylation states. Nat Genet. 2007;39:269-75.

17. Rojas AE, Ortuño D. Diferenciación celular en el Sistema Nervioso, el caso de las células precursoras neuro-gliales. Rev Bio Ciencias. 2011;1:3-17.

18. Sun W, Kim H, Moon Y. Control of neuronal migration through rostral migration stream in mice. Anat Cell Biol. 2010;43:269-79.

19. Trivedi N, Solecki DJ. Neuronal migration illuminated: A look under the hood of the living neuron. Cell Adhesion, Migration. 2011;5:42-7.

20. Kawauchi T. Regulation of cell adhesion and migration in cortical neurons: Not only Rho but also Rab family small GTPases. Small GTPases. 2011;2:36-40.

21. Khodosevich $\mathrm{K}$, Monyer $\mathrm{H}$. Signaling in migrating neurons: from molecules to networks. Front Neurosc. 2011;5:28.

22. Hernández-Miranda LR, Parnavelas JG, Chiara F. Molecules and mechanisms involved in the generation and migration of cortical interneurons. ASN Neuro. 2010;2:e00031.

23. Yamao $M$, Naoki $H$, Ishii $S$. Multi-cellular logistics of collective cell migration. PloS ONE. 2011;6:e27950. 
24. Govek EE, Hatten ME, Van Aelst L. The role of Rho GTPase proteins in CNS neuronal migration. Dev Neurobiol. 2012;71:528-53.

25. Sun Y, Huang Z, Yang K, Liu W, Xiw Y, Yuan B, et al. Self-organizing circuit assembly through spatiotemporally coordinated neuronal migration within geometric constraints. PloS ONE. 2011;6:e28156.

26. McCarthy DM, Sadri-Vakili G, Zhang X, Darnell SB, Sangrey GR, Yanagawa $Y$, et al. Cocaine alters BDNF expression and neuronal migration in the embryonic mouse forebin. J Neurosci. 2012;31:13400-11.

27. Naumburg E, Strömberg B, Kieler H. Prenatal characteristics of infants with a neuronal migration disorder: a national-based study. Int Journal Pediat. 2012;2012:541892.

28. Chatterjee CT, Sarkar CRS, Dhot BPS, Kumar LCS, Kumar CVK. Adult stem cell plasticity: Dream or reality? Med J Armed Forces India. 2010;66:56-60.

29. Viktorin G, Chiuchitu C, Rissler M, Zoltán MV, Westerfield M. Emx3 Is Required for the Differentiation of Dorsal Telencephalic Neurons. Dev Dyn. 2009;238:1984-98.

30. Pramparo T, Libiger O, Jain S, Li H, Youn YH, Hirotsune $S$ et al. Global developmental gene expression and pathway analysis of normal brain development and mouse models of human neuronal migration defects. PLoS Genet. 2011;7:e1001331.

31. Son L, et al. Human fetal brain connectome: structural network development from middle fetal stage to birth. Front Neurosci. 2017 Oct 13;11:561

32. Sousa AMM, Meyer KA, Santpere G, Gulden FO1, Sestan N. Evolution of the human nervous system function, structure, and development. Cell. 2017 Jul 13;170(2):226-247

33. Jiang $\mathrm{X}$, Nardelli J. Cellular and molecular introduction to brain development. Neurobiol Dis. 2016 Aug;92(Pt A):3-17 